\title{
Subsystems change ranking methodology (SCRaM) for complex product redesign process
}

\begin{abstract}
Product redesign is hardly a straightforward process, especially for complex products. The existence of intricate interrelationships between different components of product design architecture makes it more susceptible to change propagation phenomenon. In this case, redesign risk is not easy to predict since the change effects are being propagated to other components from the initiating change component. Because of this condition, choosing the right initiating change component is essential to control redesign process risks, apart from being able to successfully satisfy the product requirements. With this notion, this paper proposes a method that systematically ranks all components of an existing product design based on their estimated redesign risk. By having this information, designers can make a better redesign planning. The demonstration of this method is presented through an example aircraft redesign case study.
\end{abstract}

Keyword: Subsystems ranking; Product redesign; Strategic redesign; Redesign planning 